1. (pros) Aujourd'hui, je décide que le vers (octosyllabe) "L'air plus tiède arrive par bandes" est un vers-de-traduction, c'est-à-dire un vers "tourné vers". En disant "tourné vers", je parle-en-version, je verse: je pense à la préposition grecque "pros" que j'envisage comme une version (ou un original) possible du français "tourné-ver". J'ai décidé, donc, que "L'air plus tiède arrive par bandes" devenait un vers "pros". "Pros", comme la plupart des prépositions (spécialement les prépositions grecques), a plus d'un sens. Mais il a un sens premier sans doute, peut-être "tout près de", peut-être "en face". L'un et l'autre sens convient pour décrire la façon dont une version se tient par rapport à un (ou "son") original: une version, particulièrement une version interlinéaire, se tient ou se place "tout près de" ou "tout contre" l'original; une traduction se place aussi (c'est la disposition la plus courante) "en regard de" l'original, "en face", "vis-à-vis", "gegenüber" dit l'allemand: à la fois "contre” ("gegen”) et de l'autre côté ("über"). Sur la double page, lequel des deux textes regarde, surveille ou veille sur l'autre? Qui a l'œil sur qui? Le "re" du français "regarder" enveloppe plusieurs mouvements à la fois: mouvement en arrière, mouvement de répétition, mouvement en retour, retournement, inversion, conversion, etc. Le même "re" entre dans "respecter" qui est, en français, le verbe le plus fréquemment et le plus spontanément employé dans le discours sur la traduction: le traducteur "respecte" l'original, les rimes, etc. Pour "respecter", la même question se pose que pour "regarder": qui respecte qui? La traduction l'original? L'original la traduction? L'un l'autre réciproquement? Quelle est la position que tient et le mouvement que fait l'un par rapport à l'autre, dans le moment de la traduction? Est-ce qu'un seul ( le "texte d'arrivée") bouge et change? Ce sont quelques-unes des questions qui arrivent appelées par la préposition "pros", et pour lesquelles "pros" ouvre un lieu.

2. (archê) J'ai dit plus haut que le "sens premier" de "pros" était soit "vers", soit "tout près", soit "en face", soit quelque chose d'autre encore. Mais l'expression "sens premier" peut s'entendre de plusieurs façons. Elle peut désigner le sens générique ou général 
- (ARISTOTE. Métaphysique, $\Delta, 1013$ a 10 ; voir, sur archê, Aubenque, Pierre. Le problème de l'être chez Aristote. Paris: PUF, 1997 [1962]: 192-193.) dont les autres sens sont des applications particulières; le sens qui l'emporte sur les autres et fixe l'essence et les limites de la notion. "Sens premier" peut vouloir dire aussi le sens archaïque, et "sens archaïque" s'entend à son tour de deux façons, d'après les deux sens de "archê" en grec: soit le sens apparu et posé au commencement de l'histoire du mot (le commencement encore imparfait, informe et vague, de l'histoire du mot, que les sens suivants perfectionnent et quelquefois relèguent et recouvrent). Soit au contraire le sens aigu qui ne cesse pas de régir en tant que commencement jaillisant, origine, tous les autres sens envoyés par lui: "archê" au sens de ce qui n'en finit jamais de "mouvoir ce qui se meut et faire changer ce qui change", comme dit Aristote. "Si "archê" est présente et survenante de cette façon-là, alors il y a naturellement quelque chose du sens premier de "pros" dans toutes les occurrences de la préposition, et par exemple dans deux occurrences marquantes auxquelles je pense en disant que "L'air plus tiède arrive par bandes" devient un verspros. Premièrement, Aristote dit que l'être est un "pros hen legomenon" (un "dit tourné vers l'un"); deuxièmement, le premier verset de l'évangile de Jean dit que logos est "pros ton theon" (tourné vers Dieu, auprès de Dieu). Dans l'une et l'autre occurrence, il est certainement intéressant de chercher quelle position et quel mouvement sont notés par "pros"(+ accusatif). Dans le verset, la préposition exprime la médiation conduisant à l'identité ou unité: logos est tourné vers Dieu, logos est Dieu. Dans la Métaphysique, "pros" marque un point de vue sur la question "qu'est-ce que l'être?": l'être est tel que ses diverses versions se déploient ou surviennent auprès de ou tourné vers lui-même ou vers l'un. L'hypothèse que je propose, et à vrai dire l'expérience que je fais traduisant, est que, dans la relation de traduction, l'original se comporte par rapport aux versions comme l'être se comporte par rapport aux modes ou aux catégories de l'être, ou comme l'être par rapport à l'étant.

3. (vert-) La relation décrite par "pros" est réciproque, ou mutuelle. Elle l'est presque nécessairement si l'on considère l'effet ou la fonction tautologisante de " pros " dans le verset I, 1 de Jean: $x$ est pros $y$ revient à: $x$ est $y, x$ et $y$ sont le même. Mais, aussi, le verbe "être" ( $x$ est $y$ ) ne convient pas pour décrire la relation-pros, puisque l'effet de "pros" n'est pas du tout de faire être ou de fixer une essence, mais au contraire de faire devenir: deux énoncés (l'un, $x$, à partir duquel et l'autre, $y$, vers lequel se fait la traduction) deviennent "versions", ou, simplement, "deviennent". Lénoncé en langue 
étrangère que le traducteur veut faire devenir dans une autre langue, ne s'appelle "original" que par rapport au vœu du traducteur. Mais en devenant "original" par rapport au vœu, il devient lui-même ver$\operatorname{sion}^{1}$ et quitte la place de "l'original". Le nom de "version" appliqué à l'opération et à la relation de traduction, n'embrasse donc pas le même désigné que la dénomination "traduction". "Traduction" désigne l'opération, et le résultat (le texte dit "d'arrivée"). "Version" désigne l'opération et les deux énoncés qu'elle met en rapport. C'està-dire que la relation “ $x$ est $y$ ” ne veut plus dire: l'énoncé $y$ est (égal, équivalent, identique à) l'énoncé $x$, parce que l'opération de traduction a été menée à bien; " $x$ est $y$ ", considéré comme relation de version, veut dire: $x$ et $y$ ensemble (l'un et l'autre s'appelant "versions") sont "tournés vers l'un", "pros hen". Tout "se met à tourner"2: tout ce qui advient dans le moment de la version advient en "se tournant vers". Ce vers quoi tout l'advenant se tourne, c'est cela qui s'appelle "original". L'original se comporte et parle conformément à son étymologie ("orior": surgir): survenant, il fait advenir. Il est le principe ou "archê" commençant et commandant la "gravitation", le tour. Si donc je demande de quoi (de quel vers) le vers "L'air plus tiède arrive par bandes" est la version, la question maintenant se partage: d'une part, elle cherche l'énoncé (le vers), le $x$ auprès et en accompagnement duquel le vers $y$ se met à tourner; d'autre part, elle interroge sur le moteur ou "archê", c'est-à-dire $c e$ à partir ou en regard de quoi le tour tourne, le point par lequel un monde de l'être (les énoncés, textes, œuvres en tant qu’ils sont ce qu'ils sont et se tiennent dans leurs limites et dans leur essence) passe dans un monde du devenir (les œuvres s'éveillent versions).

\footnotetext{
1 "Devenir" et "verser" ("tourner": "vertere») peuvent se concevoir comme exprimant un même acte, un seul phénomène; "werden" ("devenir" en allemand) sort du même étymon que le latin "vertere".

${ }^{2}$ Le poème "Les amis inconnus" de Jules Supervielle (le premier du recueil portant aussi ce titre) commence par le vers: "Il vous naît un poisson qui se met à tourner". Il exprime (ce poème) l'humeur versatile, ou versabile, où je suis en tant que traducteur-verseur: c'est l'humeur dans laquelle toute naissance, dans le monde, me concerne, m'intér-esse (la traduction est "l'intér-essant par excellence”, dit Michel Deguy, dans "Traduire”. In: La raison poétique. Paris: Galilée, 2000: 115), où tout ce qui naît et advient, se tourne vers moi, et moi vers "il". Voir aussi le poème "Heure grave" ("Ernste Stunde") de Rilke, dans Le livre des images: "Qui va en cet instant quelque part dans le monde [...] va vers moi, etc.". C'est l'ambiance d'un "pros" universel, ou encore l'épreuve de la "versabilité infinie" dont parle Novalis, cité par Antoine Berman (L’épreuve de l'étranger. Paris: Gallimard, 1984: 31 et 125).
} 
4. (est / et) Dans la relation de version associant $x$ et $y$, aucun des deux n'est l'original. Il n'y a pas: $x$ d'abord et seul en scène, ensuite $y$ venant traduire $x$ ou venant le "relever". Il y a d'un seul coup quelque chose qui pourrait être appelé "mot-valise" (l'anglais dit: "portmanteau word"). Il n'y a pas, par exemple, d'abord "phusis" (grec), ensuite "natura" (latin) cherchant à traduire (à attirer vers soi) le premier. Dans cette conception chronologique, le premier venu dans l'histoire (en l'occurrence "phusis", dont "natura" est le calque) est soit hypostasié et soustrait à l'effort de traduction ("phusis" est "phusis", et rien d'autre), soit au contraire oblitéré: puisque "natura" est "phusis", disons désormais (dans une époque de l'histoire du monde où le latin l'emporte) "natura", et ensuite: "nature" (français, anglais), "Natur" (allemand), "natureza" (portugais), etc., de sorte que "phusis", remplacé par "natura", n'est plus du tout agissant comme archêe dans une époque de l'histoire où le grec a perdu la suprématie. Cette conception peut être appelée aussi "hegelienne": le nom par lequel un concept est nommé dans une langue et dans une époque, est relevé à l'époque suivante dans une autre langue, et "natura est phusis" se comprend comme "natura relève (relègue, annule et sauve) phusis". Une autre conception consiste à considérer que l'histoire ne produit pas du "est" (ce qui fut "phusis" est passé dans, est, "natura", et aujourd'hui sans doute dans l'anglais "nature"), ${ }^{3}$ mais du "et": et "natura" et "phusis" et encore d'autres mots (par exemple: "règne") ${ }^{4}$ ensemble annoncent et répètent quelque chose qui n'est ni présent, ni passé, ni à venir, mais qui est précisément "pros", c'est-à-dire qui est l'archê se tournant et se détournant et envoyant, en tant qu'original, des versions d'ellemême ad libitum. Il y a la traduction du côté du "est", et la version du côté du "et", il y a dans l'opération de traduction ou version la lutte que Deleuze repère dans le langage entre est et et:

Ces deux termes ne s'entendent et ne se combinent qu'en apparence, parce que l'un agit dans le langage comme une constante et forme l'échelle diatonique de la langue, tandis que l'autre met tout en variation, constituant les lignes d'un chromatisme généralisé. De l'un à l'autre, tout bascule.

\footnotetext{
${ }^{3}$ Voir la revue de langue anglaise mondialement diffusée Nature.

${ }^{4}$ C'est la version proposée (à l'écoute de Heidegger) par Bernard Sichère dans sa retraduction de la Métaphysique d'Aristote (Métaphysique, Livres A à E. Paris: Pocket, 2007.); sans doute contestera-t-on cette nouvelle version, mais, du moins, elle produit l'effet de relancer le tour, de faire faire en français un tour de plus à "phusis", au lieu de l'arrêter et de le fixer dans "nature"; elle rouvre, donc, l'espace de jeu du "et".
} 
5. (quilibet et genius et fois) Je cherche donc le vers-et, le $x$, "l'ami inconnu" associé auquel le vers "L'air plus tiède arrive par bandes" fait mot-valise, fait la paire ou rime que fend sans relâche le "est" ou original. "L'air plus tiède arrive par bandes", ce vers parle de l'arrivée du printemps. Il parle des signes par lesquels elle se fait sentir à toutes choses; à toutes choses, c'est-à-dire aussi aux choses qui annoncent ou répètent cette arrivée, qui la sont, par exemple les brises plus tièdes, le zéphyr ou "Favonius" de quelques Odes d'Horace (I, 4; IV, 7; IV 12), ou du sonnet n 310 du Canzoniere de Pétrarque, et de beaucoup de poèmes en toutes langues. Cependant, ce n'est pas du latin ni de l'italien que je traduis, mais de l'allemand. L'odelette de Mörike "Er ists" dit: "Frühling läßt sein blaues Band / wieder flattern durch die Lüfte". Dans ces deux vers, il y a "les brises" ("die Lüfte"), il y a "Band" ("ruban”, ou, homophoniquement, "bande"), il y a l'arrivée du printemps. Les deux vers de Mörike sont-ils le $x$ que je cherche? Je les traduis plutôt en disant: "Déjà le printemps ruban bleu / dans l'air flotte"; mais pourquoi le vers-version "L'air plus tiède arrive par bandes" ne s'associerait-il pas à plusieurs $x$ ? Il s'éveille version, cela veut dire: il se met à tourner en vue de cela qui fonde le tour, l'archê. L'archê ne peut pas être un autre vers particulier, mais c'est cela vers quoi se tournent les étants à l'instant où ils adviennent. Le vers-version prend en vue ou comprend en naissant ce vers-ci, et cet autre, et cet autre encore etc. Il tourne avec ces autres, mais au regard de ce qui fait tourner le tour, au regard du Devenir lui-même, tous ces vers entrant dans le tour sont des vers quelconques, des "quilibet". La comunità que viene de Giorgio Agamben commence par la phrase: "L'essere che viene è l'essere qualunque", "l'être qui vient est l'être quelconque". Si le vers-version survient au regard de et appelé par l'archêe (l'original, l'être), il se présente comme "ce quelconque" ("tode ti") ${ }^{5}$ au milieu d'autres quelconques survenant tous afin d'annoncer et de répéter ce au regard de quoi il sont tous quelconques et égaux. "Quelconque" ne veut pas dire: compris dans un

\footnotetext{
${ }^{5}$ Le "tode ti", d'Aristote, par exemple dans Catégories, 3b: "En ce qui concerne les substances premières [par exemple: l'homme individuel, le cheval individuel], il est incontestablement vrai qu'elles signifient un être déterminé [tode ti], car la chose exprimée est un individu et une unité numérique" (trad. de J. Tricot, Vrin, 1994); Frédérique Ildefonse et Jean Lallot disent (Points Seuil, 2002) "un ceci". "Tode" ("hode") est le démonstratif correspondant au latin "hic"; "ti" est un pronom indéfini ("quelque chose", "quid"). La version est ainsi au regard de l'original quelconque et singulière, elle ne se montre, ici et proche ("tode"), que pour montrer l'original.
}

'(AGAMBEN, Giorgio. La comunità che viene, Bollati Boringhieri, Torino, 2001, p. 9.) 
(AGAMBEN, Giorgio, Genius, nottetempo, 2004: 10.) genre et équivalents à tous les éléments de ce genre. Regarder un vers comme vers-version, c'est au contraire le considérer dans sa singularité insubstituable, dans son "plus propre" et dans son "genius", si le "genius" est défini comme le lieu "où le plus propre est le plus étranger et impersonnel, le plus voisin le plus éloigné et le plus inappropriable".* À partir de son lieu tout à la fois quelconque et "génial", le vers-version prend en vue l'original en tant qu'il est l'un, c'est-à-dire en tant qu'il rassemble et fait survenir tous les vers en un seul. En se présentant comme version, le vers $y$ ("L'air plus tiède arrive par bandes") cherche à atteindre dans $x$ ( $x$ étant le vers auquel il s'associe pour faire "vers-valise") le lieu originel ou "genius" à partir duquel et tourné vers lequel $x, y$ et tous les autres surviennent comme vers quelconques. Traduire au sens de la version, ce n'est pas traduire tel énoncé (tel vers, par exemple), mais chercher à répéter la fois, ${ }^{6}$ le tour ou l'original en tant qu'il est le vers des vers, c'est-à-dire le vers auprès ("pros") duquel les autres se disent. Le vers-version répète ou célèbre l'original en faisant survenir avec lui-même toujours plus de versions, c'est-à-dire en se présentant comme concomitant du plus grand nombre de vers possibles (d'Horace, Pétrarque, Mörike, etc.) et en désignant de cette façon toujours plus exactement l'original comme le lieu où s'origine le tour. Et réciproquement, plus l'original est original, plus il appelle à sa place des versions diverses; il se reconnaît à ceci qu'il se retire à chaque tour de la place de l'un, mandant à sa place toujours quelque autre version, et que, comme la nature, "il aime se cacher"; la version est le moyen par lequel l'original devient toujours plus l'original.

6. (synesthésie) Chercher "l'original" de $y$, c'est donc chercher, parmi les $x$ appelés à la fête des versions (la fête célébrant l'original), l'invité le plus proche, c'est-à-dire la plus proche version à l'instant de la survenue de $y$. "Laue Luft kommt blau geflossen" est le premier vers du poème "Frische Fahrt" de Eichendorff. Je décide que c'est vers lui que se tourne, en survenant, "L'air plus tiède arrive par bandes", et que les deux vers, le français et l'allemand, sont concomitants, c'est-à-dire qu'ils vont ensemble s'accompagnant l'un l'autre. La concomitance indique que $x$ ne précède pas $y$ (l'allemand le français), mais que d'emblée ils font mot-valise, rime, c'est-à-di-

\footnotetext{
6 "Volta", "vez", "fois": en italien, portugais, espagnol, français, l'idée de la "fois", c'est-à-dire du mouvement de temps par lequel quelque chose agit entéléchiquement et cède la place, se dit par le "tour".
} 
re une unité césurée. Le même phénomène de concomitance césurée se montre dans l'expression "alter ego" et plus encore peutêtre dans la formulation grecque "heteros autos"; Agamben, dans L'amitié, lisant et traduisant un passage du livre IX de l'Éthique à Nicomaque d'Aristote, et en particulier l'expression "heteros autos" (Nic. 1170 b) comparée à “alter ego", écrit ceci:

le latin ego ne correspond pas exactement à autos, qui signifie "soimême”. L'ami n'est pas un autre moi, mais une altérité immanente dans la mêmeté, un devenir autre du même. Au point où je perçois mon existence comme douce, ma sensation est traversée par un consentir qui la disloque et la déporte vers l'ami, vers l'autre même.

Ce qui, dans la citation ci-dessus, s'appelle "con-sentir" dans la traduction de l'italien d'Agamben, correspond au "sunaisthanomai" d'Aristote, au "sentir-avec", "sentir-partagé"; à l'instant de la sensation douce de se partager, les deux vers amis (l'allemand et le français) vivent ensemble ("suzên", Nic. 1170 b), partageant un même, ne partageant rien d'autre que le "sun-". L'amitié, dit Agamben,

est le partage qui précède tout autre partage, parce que ce qu'elle départage est le fait même d'exister, la vie même.

C'est de ce partage, qui n'est pas le partage de quelque chose, que vit la relation de version. "Alter" et "ego", $x$ et $y$, tournés l'un vers l'autre, constituent en tant que paire ou que "centaure", un seul signe concomitant tourné vers la césure (l’original) les départageant et les constituant en deux "natures" distinctes, comme les centaures sont dits aussi "de nature double" ("diphuês"). Le redoublement de "cum" qui se remarque dans le verbe concomitor (cum-cum-ire) convient à décrire la relation de version: $x$ et $y$, en effet, accompagnent l'accompagnant; ils vont à deux comme les deux disciples sur la route d'Emmaüs, mais leur "comitance" ne trouve son sens que par la survenue entre eux d'un troisième ( $L u c, 24,15$ et suivants). À l'instant même où, le reconnaissant, ils sont capables de l'appeler par son nom, le tiers (le Christ) devient invisible. Cet instant de la reconnaissance et du partage (les disciples sont ou deviennent des chrétiens, c'est-à-dire s'appellent du nom du tiers), c'est toujours l'instant 0 de la version, où le tour commence. Les versions, alter et ego, partagent l'original en tant qu'il est un nom.

7. (vix et acteur et hamartia) Quel est le nom propre que partagent le premier vers de "Frische Fahrt" de Eichendorff, et la version française qui me vient? Qu’est-ce qui césure la rencontre? Est-
* (AGAMBEN, Giorgio. L'amitié. Trad. de l'italien par Martin Rueff. Paris: Rivages poche, 2007: 34-35.)

(Ibidem: 40.) 
- (DELEUZE, Gilles. Différence et répétition. Paris: PUF, 2000: 54.) ce "lau" ("tiède")? "kommen" ("venir")? "fließen" ("couler")? "fahren" ("aller", dans le titre), "Frühling" ("printemps", dans le vers suivant), etc.? Mais si c'était un seul nom que $x$ et $y$ partageaient, ce serait l'économie de l'équivalence, de l'échange et de la généralité, et le partage ne serait pas gracieux comme dans l'amitié. C'est toujours au contraire une série (par exemple la série de mots que je viens d'énumérer) et un tour, et le nom recherché est toujours une variable qui s'appelle, de tour en tour, d'une nom chaque fois différent. Le poème $x$ (dit "de départ" dans la relation de traduction, que j'oppose à celle de version) ne devrait pas s'appeler "version", s'il ne se conduisait pas, en lui-même, selon le point de vue de la version, s'il ne tournait pas lui-même en vue et auprès de l'archê mobilisante. Dans le poème même $(x)$, tout s'appelle du nom recherché, ou, réciproquement: la variable hantant le poème prend tour à tour tous les noms (adjectifs, verbes, pronoms, etc.) sur la ligne desquels le poème se déplie. Le nom propre (variable, archê, original) est le tour ("vix") relevant à chaque fois les vicissitudes (c'està-dire le sens se déployant de moment en moment, syntaxiquement, rythmiquement, etc.) du poème. Le nom propre ou variable n'accomplit aucun autre acte dans le poème que l'acte de céder la place, ${ }^{7}$ de "vicarier", et le poème n'est à son tour que l'arrangement en série des vicaires du nom propre, chacun se faisant prendre à son tour pour le nom original ou "puissance invitante", avant de s'avérer tout de suite invité au lieu d'hôte invitant. Ce n'est pas un système hiérarchique. Ce n'est pas l'enveloppement de particuliers au sein d'espèces, ou d'espèces au sein de genres. C'est plutôt une répartition anarchique ou "nomadique", pareille à la distribution de "l'être univoque"; c'est, selon les termes de Deleuze,

une distribution d'errance et même de "délire", où les choses se déploient sur toute l'étendue d'un Être univoque et non partagé. Ce n'est pas l'être qui se partage d'après les exigences de la représentation, mais toutes choses qui se répartissent en lui dans l'univocité de la simple présence (l'Un-Tout).

Le poème tournant dans l'univocité du tour se relève sauf, "intact”; il peut dire "j' avais cessé de me sentir médiocre, contingent, mortel”, comme écrit Proust décrivant la joie liée au goût de la Petite Madeleine trempée. La version, c'est-à-dire la joie de l'un, a

\footnotetext{
${ }^{7} \mathrm{La}$ fois ou archê du poème se décrit comme la "place toujours cédée"; le mot latin "vix" (inusité au nominatif, cf. "vice versa") signifie d'abord la "place", il est apparenté à l'allemand "weichen", "céder", "se retirer".
} 
toujours déjà commencé à même le poème $x$. D'ailleurs, il ne pourrait devenir l'hôte d'aucune version $(y)$ s'il ne se déployait lui-même en tant que tour et devenir. La version $(y)$, maintenant, ne fait que doubler, mimer ou jouer le tour, ou la tournure prise déjà par $x$. Elle "est" $x$, au sens où l'acteur est le personnage (où Laurence Olivier est Hamlet). Hamlet, à son tour, qu'est-il? Que veut-il et que désigne-t-il? Acteur et "personnage" désignent le "même". Ils sont l'un et l'autre acteurs de l'événement, de la fois. Le vers "L'air plus tiède arrive par bandes" désigne l'original, le tour lui-même. Voulant désigner l'original, il touche nécessairement à côté, puisque l'original n'est rien d'autre que la place sans relâche vidée et cédée. Voulant désigner à l'instant même l'original, je désigne la version la plus proche à cet instant. Ma version n'est pas équivalente (par exemple, "par bandes" n'est pas "geflossen"). Mais l'écart (au regard de $x$ ) court la chance d'être relevé au regard de l'original lui-même. La visée errante, le péché ${ }^{8} \mathrm{~d}^{\prime}$ infidélité, s'interprètent éventuellement comme envoyés et voulus par le lieu lui-même, ou tour, ou point, d'où le poème sort. Fahrt":

8. (Ort et éthique) Voici les deux premiers vers de "Frische

Laue Luft kommt blau geflossen

Frühling, Frühling soll es sein!

Voici la traduction que je présente:

l'air plus tiède arrive par bandes

le printemps me rencontre-t-il

J'ai indiqué plus haut $(\$ 5)$ que le premier vers de ma version se détournait certainement du seul vers de Eichendorff afin de se tourner aussi vers le vers de Mörike "Frühling läßt sein blaues Band", c'est-à-dire qu'il voulait appeler et convertir plus d'un vers en un, et, par ce tour, visait à faire venir le poème lui-même ("Frische Fahrt") comme le poème du devenir, le tour lui-même: c'est l'ambition de toute version de se tourner vers le poème étranger de façon critique, c'est-à-dire de telle sorte qu'il entrerait en présence comme le poème des poèmes, l'un-tout et le quelconque. Le deuxième vers, "le printemps me rencontre-t-il", se tourne peutêtre à son tour vers quelque chose d'Apollinaire, peut-être le pre-

\footnotetext{
8 "Peccatum" ("péché") de la Vulgate est la traduction du grec "hamartia", issu du verbe "hamartanô", "manquer le but".
} 
mier poème de Vitam impendere amori ("Te souviens-tu de sa rencontre [...] / Encore un printemps de passé") peut-être l'aubade de "La chanson du mal-aimé". La version a pris la parole et emporté l'autre poème dans son tour, sans pourtant présenter rien d'équivalent à ce que l'autre présente. Les deux vers allemands (et tout le poème) sont des tétramètres trochaïques. Ponctués d'allitérations et d'assonances (Lau-luft-blau-floss, omm-oss; f-f; s-s), ils appartiennent à un type fréquent chez Eichendorff: le type du "Stabreimvers", le vers allitérant de la poésie germanique originelle. Le traducteur-verseur n'estime pas que la valeur ("Wert") du poème tient à ces traits (allitérations, etc.) et qu'il devrait miser sur des "trouvailles" (par exemple des allitérations équivalentes) comme étant la seule monnaie d'échange lui donnant le droit d'emporter ou d'empocher le poème à traduire. Les deux premiers vers français sont mus au contraire par une sorte de travail d'aversion, éloignant d'emblée $y$ de $x$, comme si le travail de version devait consister ensuite à combler l'écart, à rentrer vers $x$ quitté dès l'abord et à "demander pardon" à ce qui a été trahi. Mais c'est une sorte de fidélité aussi qui commande de s'écarter. Il y a un poème de Hölderlin (l'hymne "Die Wanderung", "La migration") dont le thème est cette infidélité-fidélité, ce mouvement d'aversion, de migration lointaine et de retour. Dans ce poème, le poète est "enfant de la Souabe", et la Souabe est dans la position du poème-à-traduire; chacun de ses traits est aimable et "nulle part il ne fait meilleur vivre" (et dans l'ensemble, elle est comparable à la Lombardie voisine). Mais son trait le plus remarquable est sa fidélité à l'origine (“Ursprung") ou encore "lieu" (“Ort"):

c'est pourquoi

t'est innée la fidélité. Difficilement quitte

ce qui près de l'origine [Ursprung] habite, le lieu [Ort].

Dans le dernier vers cité, "Ort" à la fin du vers, au "bout" ("bout", "pointe" sont les sens premiers de l'allemand "Ort"), arrive comme version de "Ursprung", ou inversement. La même chose (peut-être la même) s'appelle tour à tour de deux noms. La langue se déplace et change auprès de l'un, "pros hen". Deux noms

\footnotetext{
${ }^{9}$ Il y a une étude à consacrer à la notion de "trouvaille" et à la question de savoir pourquoi elle est limitée apparemment à la description du travail du traducteur (peut-être Valéry parle-t-il de "trouvaille" à propos de l'élaboration d'un poème); sur la "trouvaille", voir Marc de Launay (Qu'est-ce que traduire. Paris: VRIN, 2006, p. 50).
} 
se cèdent le place, le tour. Le second pourrait être rendu par "le point”. La Souabe n'est pas d'abord aimable pour ses splendeurs (arbres, rivières, villes), mais par le point auprès duquel elles tournent. Considérée comme poème, la Souabe se constitue avant tout comme fidélité à "Ort”, comme contrée où tour à tour chacun des arbres, chacune des villes, etc. se tourne vers le seul "lieu" et la seule "fois". Le poète de la Souabe (Hölderlin) est le traducteur, c'est-àdire le "verseur", de son pays natal. Verser et chérir le pays natal ne veut pas dire en observer et en reproduire les traits adorables. Cela veut dire au contraire s'en détourner, ne pas le voir:

Mais moi je veux me tourner vers le Caucase!

(premier vers de la troisième strophe du poème). L'origine exige l'aversion ou infidélité. L'infidélité du poète-verseur (voyageur) répond à la fidélité du poème à traduire (la Souabe), dans la mesure où la fidélité de la Souabe était tout entière tournée vers le point ("Ort"). Ce n'est pas une fidélité visant à reproduire "trait pour trait", c’est une fidélité au nom, "Ort”, pour autant qu'il ne désigne rien que de l'invisible quelconque et ne fait rien d'autre dans le poème (dans la contrée) que céder sans relâche la place à ses avatars ou vicaires. C'est une position éthique au sens ou Emmanuel Levinas dit que

l'accès au visage est d'emblée éthique. C'est lorsque vous voyez un nez, des yeux, un front, un menton, et que vous pouvez les décrire, que vous vous tournez vers autrui comme vers un objet. La meilleure manière de rencontrer autrui, c'est de ne pas même remarquer la couleur de ses yeux! Quand on observe la couleur des yeux, on n'est pas en relation sociale avec autrui. La relation avec le visage peut certes être dominée par la perception, mais ce qui est spécifiquement visage, c'est ce qui ne s'y réduit pas.

9. (forma, version interlinéaire, dire) Ce qui ne se réduit pas à la perception, c'est "Ort” dont le nom désigne l'imperceptible même, et la meilleure manière d'être tourné vers le visage de la Souabe est de le rencontrer dans et par "Ort". Accès éthique: il faudrait ne pas même remarquer les allitérations et autres phénomènes remarquables ("vains ornements") dans les deux premiers vers de "Frische Fahrt”. Ce n'est pas de l'admiration fascinée pour la forme perceptible ("forma") de $x$ que sort le désir de version. L'humeur versatile s'intéresse à $x$ en tant qu'il se tourne et change, et non pas en tant qu'il se tient et pose; $x$, en tant qu'il est apte-à-verser et lui-même versatile, ne se montre que montrant le point, "Ort". L'attention
' (LEVINAS, Emmanuel. Éthique et infini. Paris: Livre de Poche, 1988: 79-80.) 
(Ibidem: 80.)

(Ibidem: 82.)

- (LEVINAS, Emmanuel. Autrement qu'être ou au-delà de l'essence. Paris: Livre de poche, 1996 [1978]: 17.) et la fidélité versatiles vont à ce qui est tourné vers soi-même seulement en tant que point, et à ce qui, en tant que texte-à-verser, se tourne sans relâche vers autre que lui-même, comme la Souabe vers “Ort". D'un côté comme de l'autre, $x$ ne se présente donc pas paré de beautés, mais dans une "pauvreté essentielle", comme dit Levinas à propos de l'exposition du visage d'autrui et de son dénuement. ${ }^{*}$ La présentation bilingue sur la double page où le poème étranger prend la pose sur la page de gauche est trompeuse, de ce point de vue: soit elle exprime que l'original est irrémédiablement premier et inaccessible et ne pourra plus jamais, en tant que commencement, être renversé, versé; soit elle indique au contraire que $x$ et $y$ placés en vis-à-vis se rangent dans un Même et sous un seul genre, intégration à partir de laquelle il est devenu possible justement de faire passer $x$ dans $y$, c'est-à-dire d'élaborer un équivalent. Le vis-à-vis bilingue rapporte donc éventuellement comment $x$ a été pris en vue, puis pris et déduit: tra-duit. Au contraire, la disposition interlinéaire - ce discours myope où le texte étranger est rapproché au point que plus rien de cohérent et de formel n'est vu de lui - interdit la prise et la compréhension de $x$ et de $y$ dans un même genre (interdit la "totalisation”). La version interlinéaire s'aventure donc comme réponse immédiate apportée d'un coup au texte-à-verser, comme le "dire" précédant le "dit":

le dire, c'est le fait que devant le visage je ne reste pas simplement là à le contempler, je lui réponds. Le dire est une manière de saluer autrui, mais saluer autrui, c'est déjà répondre de lui. Il est difficile de se taire en présence de quelqu'un $[\ldots]^{*}$

L'humeur versatile, dans la mesure où elle advient comme l'autre de l'émulation concurrente et équivalente, advient dans le "dire originel ou pré-originel - le propos de l'avant-propos" et "noue une intrigue de responsabilité". De même que l'analyse du poème de départ précède la traduction à visée d'équivalence, de même la version interlinéaire arrive comme une préface ouvrant le lieu de la version et annonçant la vacance du tour; version, ou: autrement qu'être, être comme fois, détour et avent. Voici "Frische Fahrt" accompagné d'une version interlinéaire:

Frische Fahrt

fraîche (vive, neuve) excursion

Laue Luft kommt blau geflossen, tiède air vient bleu coulant (par vagues) 
Frühling, Frühling soll es sein!

printemps printemps doit (dit-on) cela être

Waldwärts Hörnerklang geschossen,

vers-forêt son-des-cors tiré

Mut'ger Augen lichter Schein;

(de) courageux yeux (le) clair éclat

und das Wirren bunt und bunter

et le fouillis désordonné-multicolore et plus désordonné-multicolore

Wird ein magisch wilder Fluß

devient un magiquement sauvage fleuve

In die schöne Welt hinunter

dans le beau monde vers-le-bas

Lockt dich dieses Stromes Gruß.

attire t' de-ce fleuve (courant) le-salut

Und ich mag mich nicht bewahren!

Et je peux (pourrais) moi ne pas prouver-ma-valeur

Weit von euch treibt mich der Wind

Loin de vous entraîne moi le vent

Auf dem Strome will ich fahren,

Sur le fleuve veux moi voyager

Von dem Glanze selig blind!

De l' éclat bienheureusement aveugle

Tausend Stimmen lockend schlagen,

mille voix attirant éclatent

Hoch Aurora flammend weht,

haut Aurore flamboyant souffle

Fahre zu! ich mag nicht fragen,

Va encore je veux ne-pas demander

Wo die Fahrt zu Ende geht!

où le voyage à (la) fin va

10. (métamorphoses, etc.) Comme "Er ists" de Mörike, "Frische Fahrt" parle (comme beaucoup d'autres poèmes de Eichendorff) de la venue du printemps dans une ambiance d'incrédulité (joyeuse ou triste); il y a toujours doute sur la survenue du printemps, du "prime time", de la première fois, de la "fois pour toutes". Avec ("pros") le printemps, tout advient, devient: "kommt blau geflossen" (v. 1); l'azur lui-même et les brises bleues sont montrés comme "ce qui vient azurant". Le premier vers contient (comme tout poème sur la venue du printemps peut-être) une espèce de "jam" (déjà, à nouveau, bientôt, déjà plus). L'adverbe latin "jam” est une espèce de démonstratif montrant le temps, c'est-à-dire la fois, et donc montrant toujours nécessairement à côté et se trompant, montrant la place toujours déjà vidée où advient et devient un avatar de la "fois". Le printemps venant sur le mode de "jam” (peut-être est-il déjà parti, 
ou encore arrivant), tout dans le monde se met à tourner-devenir: versabilité universelle. Eichendorff a un verbe pour exprimer cela, son verbe-clef (absent de "Frische Fahrt"): "wandern" (itératif de "winden", "tourner", "tresser", $c f$. l'anglais "wander"), "se mettre en route et marcher". Dans "Frische Fahrt", le voyager se dit par "fahren" (voyager en bateau, voiture, etc.), parce que c'est la descente d'un fleuve dont il s'agit; cela tient aux circonstances romanesques dans lesquelles le poème intervient (le poème est chanté par un personnage du roman Ahnung und Gegenwart, Pressentiment et présent, livre II, chap. 12). Mais au regard de la circonstance poétique, "fahren" se présente ici comme un avatar ou un lieutenant de "wandern", et "wandern" lui-même comme la version interlinéaire ou la contrepartie d'un autre verbe allemand, "wandeln", qui peut servir à exprimer la venue et la marche des dieux sur terre, et qui veut dire aussi "changer". "Wandern" désigne une espèce d'"acte-valise" par lequel les hommes se changeant universellement en voyageurs correspondent à la venue sur terre des dieux, c'est-à-dire à la survenue du printemps, c'est-à-dire de la fois, ou du temps manifeste. Cherchant à traduire (verser) "wandern" qu'on trouve tout le temps dans les poèmes de Eichendorff, je dis en français: voyager $e t$ vagabonder $e t$ randonner $e t$ errer $e t$ marcher $e t$ aller $e t$ arriver $e t$ se produire et vaguer $e t$ être envoyé $e t$ commencer $e t$ tourner et devenir etc. Ce n'est pas du tout que l'équivalent differe selon le contexte. C'est bien plutôt qu'à chaque fois le "verseur" veut tourner d'un coup "wandern" comme une seule fois, tous les événements-wandern comme un seul événement. C'est ce désir tourné vers l'un qui reçoit à chaque coup sa punition-récompense dans un nouvel avatar, un nouveau tour, donné et joué, et entrant dans la ronde ${ }^{10}$ - une nouvelle métamorphose, en entendant "métamorphose" comme Michel Deguy:

par la traduction, une chose (en l'occurrence une "œuvre" sous les espèces d'un "texte") peut devenir; devient indéfiniment n'importe quoi d'autre en ne cessant pas d'être "la même", liée à son origine fictive, ou "original".

11. (tourner, délabrer) Ma version française commence par deux octosyllabes, et lance ou fait attendre un système de rimes alternativement féminines et masculines. Mais le système se détra-

${ }^{10}$ Comme tous les dieux (comme Dionysos en particulier), "wandern" reçoit tous les noms et du moins plusieurs noms, et le "nom véritable" est l'archê ou le tour envoyant tous les autres. 
que dès le v. 4, heptasyllabe et non-masculin. Ce qu'il y a à faire ne relève pas du système. Ce que le "tourneur" a sur les bras, à chaque moment, c'est la tâche de se tourner vers le point. La version mutile son propre visage, mais elle doit être encore assez visage et forme pour désigner ce qui est à désigner (le visage ne se fait voir que pour faire voir autre que lui-même), et sa poétique est pour cette raison souvent voisine de la poétique "délabrée" de la chanson, où la rime devient assonance et où le mètre pèche par excès ou par défaut. Voici ma version de "Frische Fahrt", déjà se tournant et défaisant:

Départ

l'air plus tiède arrive par bandes le printemps me rencontre-t-il des bois partent des cors de chasse l'ardeur dans les yeux pétille et toujours plus le clair tumulte par vague magique sauvage le monde est beau descends ce fleuve de la vie t'appelle

et qui ne voudrait s'éprouver loin de vous le vent m'entraîne je veux descendre le fleuve un rayon pur bonheur m'aveugle mille voix séductrices claquent de flamme est le vent et voyage je ne demande pas où tu vas

\section{Philippe Marty}

Agrégé de alemão, é professor de literatura geral e comparada da Universidade de Nice Sophia Antipolis (France). Sua tese de doutorado teve por tema a tradução de poesia e sua tese de habilitação foi publicada (Le poème et le phénomène. Lectures de noms propres. Éditions Le Manuscrit, 2007). Tem diversos artigos sobre poesia, tradução e a relação entre filosofia e poesia, em torno de autores como Sófocles, Petrarca, Valéry, Rilke, Bobrowski. É ainda tradutor de Hölderlin, Friedrich Schlegel e de Eichendorff (no prelo), pela editora Grèges (Montpellier, França), e de Georg Simmel, pela Maison des Sciences de l'Homme, Paris. 
Palavras-chave: archê; versão; verter; vertibilidade; original; ética; devir.
Key words: archê; version, translation; translatability; original; ethics; becoming.

Mots-clés: archê; version; verser; versabilité; original; éthique; devenir.

\section{Resumo}

Neste ensaio, a palavra "tradução" cede aos poucos seu lugar à palavra "versão". A grande diferença entre elas é que a palavra "tradução" só pode servir para designar o que se diz na chegada, na língua em que se traduz; ao passo que a palavra "versão" pode expressar ao mesmo tempo o original em língua estrangeira e o que dele decorre na língua do tradutor. Pois o original é descrito aqui como o que, a cada vez, a cada esforço feito para atingi-lo e encontrá-lo, cede lugar, retira-se, e indica sempre o que concerne simultaneamente ao texto de partida e ao texto de chegada e que é a fonte e o fim de toda tradução, ou versão. No mundo da versão, tudo "começa a girar", como diz um verso de Jules Supervielle. É deste "humor versátil" que este estudo quer fazer a descrição, passando por Aristóteles, Deleuze, Levinas e Agamben, tomando como exemplo um poema de Eichendorff e, mais precisamente, o primeiro verso do poema sobre o retorno da primavera. Animado, porém, pelo humor versátil, o tradutor deseja traduzir em um só verso todos os versos do mundo.

\section{Abstract}

In this essay the word "translation" is gradually replaced by "version". The great difference between them is that "translation" can only function to designate what is meant in the target language one is translating into, whereas "version" may express both the original in the foreign language and the end result in the translator's native tongue. For the original is here described as that which, at each attempt to reach it, to find it, withdraws, becomes vacant, and always indicates what concerns simultaneously the original text and the final one, and which is the source and end of any translation or version. In the world

\section{Résumé}

Dans cette étude, le mot "traduction" cède la place peu à peu au mot "version". La grande différence entre les deux est que le mot "traduction" ne peut servir qu'à désigner ce qui se dit à l'arrivée, dans la langue où l'on traduit; alors que le mot "version" peut exprimer d'un coup et l'original en langue étrangère et ce qui découle de lui dans la langue du traducteur. Car l'original est décrit ici comme ce qui, à chaque fois, à chaque effort fait pour l'atteindre et le rencontrer, cède la place, se retire et indique toujours ce qui regarde à la fois le texte de départ et le texte d'arrivée et qui est la source et la fin de toute traduction, 
of versions, everything "starts to rotate", as in a verse of Jules Supervielle. It is this "versatile humor" that this study intends to describe, dealing with Aristotle, Deleuze, Levinas and Agamben, taking a poem of Eichendorff as example, more precisely its first line about the return of spring. Animated, however, by versatile humor, the translator wishes to translate all the verses of the world into one single line. ou version. Dans le monde de la version, tout "se met à tourner", comme il est dit dans un vers de Jules Supervielle. C'est cette "humeur versatile" dont cette étude veut faire la description, passant par Aristote, Deleuze, Levinas et Agamben, l'exemple pris étant un poème de Eichendorff, et plus précisément le premier vers de ce poème sur le retour du printemps. Mais, animé par l'humeur versatile, le traducteur désire traduire dans un seul vers tous les vers du monde. 